\title{
Frequency and Impact of Intensive Care Unit Complications on Moderate-Severe Traumatic Brain Injury - Early Results of the Outcome Prognostication in Traumatic Brain Injury (OPTIMISM) Study
}

Susanne Muehlschlegel, MD, MPH ${ }^{1,2,3}$; Raphael Carandang, $\mathrm{MD}^{1,3}$; Cynthia Ouillette, $\mathrm{RN}^{1}$, Wiley Hall, $\mathrm{MD}^{1,3}$; Fred Anderson, $\mathrm{PhD}^{3,4}$; Robert Goldberg, $\mathrm{PhD}^{5}$

${ }^{1}$ Departments of Neurology, Division of Neurocritical Care, ${ }^{2}$ Anesthesia/Critical Care and

${ }^{3}$ Surgery, ${ }^{4}$ Center for Outcomes Research, ${ }^{5}$ Department of Quantitative Health Sciences;

University of Massachusetts Medical School, Worcester, MA

Contact information:

Susanne Muehlschlegel, MD, MPH

Assistant Professor of Neurocritical Care

Departments of Neurology (Division of Neurocritical Care), Anesthesiology/Critical Care and

Surgery

University of Massachusetts Medical School

55 Lake Ave North

Worcester, MA 01655

Phone: 508-334-4651

FAX: 508-856-6778

Email: susanne.muehlschlegel@umassmemorial.org

\begin{abstract}
Background: Known predictors of adverse outcomes in patients with moderate-severe TBI (msTBI) explain only a relatively small proportion of patient-related outcomes. The frequency and impact of intensive care unit complications (ICU-COMPL) on msTBI-associated outcomes is poorly understood.

Methods: In 213 consecutive msTBI patients admitted to a Level-I-Trauma-Center neurotrauma-ICU, twenty-eight ICU-COMPL (21 medical and 7 neurological) were prospectively collected and adjudicated by group consensus, using pre-defined criteria. We determined frequencies, and explored associations of ICU-COMPL and functional neurological outcomes measured by Glasgow Outcome Scale (GOS) at hospital discharge using multivariable logistic regression.
\end{abstract}

Results: The average age of the study sample was 53 years, and the median presenting Glasgow Coma Scale and Injury Severity Scores were 5 and 27, respectively. Hyperglycemia (79\%), fever (62\%), systemic inflammatory response syndrome (60\%), and hypotension requiring vasopressors (42\%) were the four most common medical ICU-COMPL. Herniation (39\%), intracranial rebleed (39\%), and brain edema requiring osmotherapy (37\%) were the three most common neurological ICU-COMPL. After adjusting for admission variables, duration of ventilation, and ICU length-of-stay, patients with brain edema (OR 5.8; 95\% CI 2,16.7) had a significantly increased odds for dying during hospitalization whereas patients with hospitalacquired urinary tract infection (UTI) had a decreased odds (OR 0.05; 95\% Cl 0.005,0.6). Sensitivity-analysis revealed that UTI occurred later, suggesting a non-causal association with survival. Brain herniation (OR 15.7; 95\% Cl 2.6,95.4) was associated with an unfavorable functional status (GOS 1-3).

Conclusion: ICU-COMPL are very common after msTBI, have a considerable impact on shortterm outcomes, and should be considered in the prognostication of these high-risk patients. Survival associations of time-dependent complications warrant cautious interpretation. 
